\title{
Tradução e Validação do Movement Imagery Questionnaire - 3 (MIQ - 3) com Atletas Portugueses
}

\author{
Translation and Validation of the Movement Imagery Questionnaire - 3 \\ (MIQ - 3) with Portuguese Athletes
}

\author{
Pedro Alexandre Mendes ${ }^{1}$, Daniel A. Marinho ${ }^{2,3}$, João Duarte Petrica ${ }^{1,4}$, Paulo Silveira ${ }^{1}$, \\ Diogo Monteiro ${ }^{5}$, Luis Cid ${ }^{2,5}$
}

ARTIGO ORIGINAL | ORIGINAL ARTICLE

\begin{abstract}
Imageryé um processo cognitivo que pode desempenhar um papel importante no planeamento e execução de movimentos ou ações. Vários instrumentos têm sido desenvolvidos com o intuito de avaliar a habilidade de Imagery no desporto. No entanto, nenhum se focou na modalidade cinestésica, visual interna e visual externa. O objetivo deste estudo foi traduzir e validar preliminarmente para a população desportiva portuguesa o Movement Imagery Questionnaire-3, determinando as suas qualidades psicométricas iniciais através de uma análise fatorial exploratória do modelo que a suporta. A amostra foi constituída por 176 sujeitos $(\mathrm{N}=176)$ (sexo masculino $\mathrm{N}=134$; sexo feminino $\mathrm{N}=42$ ) com uma média de idades de 20.08 anos $(\mathrm{SD}=5.94)$, composta por atletas de futebol $(\mathrm{N}=113)$, basquetebol $(\mathrm{N}=43)$ e ténis $(\mathrm{N}=20)$. Numa primeira fase verificou-se que os procedimentos de tradução e adaptação originaram uma versão Portuguesa do MIQ - 3 semelhante à versão original. Numa segunda fase verificamos que as qualidades psicométricas comprovam a adequação da adaptação efetuada, demonstrando que a sua estrutura fatorial é igual à versão original (12 itens agrupados em 3 fatores, com 4 itens cada fator), apresentando índices bastante aceitáveis de validade e fiabilidade (alfa de Cronbach: 0.88 para MIQ - 3, 0.79 para as três modalidades), concluindo que este questionário poderá ser utilizado para avaliar a capacidade de Imagery em atletas Portugueses.

Palavras-chave: análise fatorial exploratória, desporto, imagery, tradução e validação
\end{abstract}

\begin{abstract}
Imagery is a cognitive process that can play an important role in planning and execution of movements or actions. Several instruments have been developed in order to evaluate the ability of Imagery in sport. However, none focused on the kinesthetic, visual internal and visual external modalities. The objective of this study was to translate and validate preliminary for the Portuguese sports population the Movement Imagery Questionnaire-3, determining its initial psychometric qualities through an exploratory factor analysis model that supports it. The sample comprised 176 subjects $(\mathrm{N}=176)(\mathrm{N}=134$ male, female $\mathrm{N}$ $=42)$ with a mean age of 20.8 years $(\mathrm{SD}=5.94)$, consisting of soccer $(\mathrm{N}=113)$, basketball $(\mathrm{N}=43)$ and tennis $(\mathrm{N}=20)$ players. Initially it was found that the procedures of translation and adaptation originated a Portuguese version MIQ - 3 similar to the original version. Secondly we found that the psychometric qualities prove their suitability of adaptation performed, demonstrating that its factor structure is the same as the original version (12 items grouped into 3 factors, with 4 items each factor), with quite acceptable levels of validity and reliability (Cronbach's alpha: 0.88 to MIQ - 3, 0.79 for the three modalities), concluding that this questionnaire can be used to assess Imagery capacity in Portuguese athletes.
\end{abstract}

Keywords: exploratory factor analysis, sport, imagery, translation and validation

\footnotetext{
Artigo recebido a 08.06.2015; Aceite a 28.07.2015

${ }^{1}$ Escola Superior de Educação, Instituto Politécnico de Castelo Branco, Castelo Branco, Portugal

${ }^{2}$ Universidade da Beira Interior, Departamento de Ciências do Desporto, UBI, Covilhã, Portugal

${ }^{3}$ Centro de Investigação em Desporto, Saúde e Desenvolvimento Humano, CIDESD, Portugal

${ }^{4}$ Centro de Estudos em Educação, Tecnologias, CI\&DETS, Viseu, Portugal

${ }^{5}$ Escola Superior de Desporto de Rio Maior, Instituto Politécnico de Santarém, Rio Maior, Portugal

* Autor correspondente: Av. Pedro Álvares Cabral, nº 12, 6000-084 Castelo Branco E-mail: pedromendes@ipcb.pt
} 


\section{INTRODUÇÃO}

Imagery é um processo cognitivo que pode desempenhar um papel importante no planeamento e execução de movimentos ou ações (Cumming \& Williams, 2013). No contexto desportivo, o Imagery pode ser considerado como uma criação ou recriação de uma experiência gerada a partir da informação da memória, envolvendo caraterísticas sensitivas, percetivas e afetivas, que podem ocorrer na ausência de estímulo real antecedente, normalmente associado com a experiência que deve proporcionar efeitos fisiológicos e psicológicos na pessoa que realiza a ação (Morris, Spittle, \& Watt, 2005). Holmes e Calmels (2008) apresentam uma noção de Imagery adaptada de Morris, Spittle, e Watt (2005): Imagery, no contexto desportivo, pode ser considerada como a geração neural ou regeneração das partes neurais, representativas da rede cerebral envolvendo, de cima para baixo, características sensoriais, percetuais e afetivas, que estão, sobretudo dependentes do controlo consciente do indivíduo e que podem ocorrer na ausência da aferência percetual e que é funcionalmente equivalente à ação desportiva visualizada. Hall (2001) sugere ainda, que o Imagery pode ser considerado uma competência ou habilidade porque pode ser melhorada através da sua prática regular e deliberada. Embora o Imagery ocorra nas diversas modalidades sensoriais (visual, auditiva e olfativa), o focus do Imagery no movimento é frequentemente no Imagery visual e cinestésico (Cumming \& Ste-Marie, 2001). A efetividade do Imagery para melhorar o desempenho depende da capacidade individual do indivíduo para gerar e controlar imagens vívidas (Martin, Moritz, \& Hall, 1999). Vários instrumentos têm sido desenvolvidos com o intuito de avaliar a habilidade de Imagery no desporto, como o Vividness of Movement Imagery Questionnaire (VIMQ), de Issac, Marks, e Russel (1986), Sport Imagery Questionnaire (SIQ), de Hall, Mack, Paivio, e Hausenblas (1998) e Movement Imagery Questionnaire (MIQ-R), de Hall e Martin (1997). No entanto, nenhum se focou na modalidade cinestésica, visual interna e visual externa. O Movement
Imagery Questionnaire (MIQ) foi especificamente projetado para avaliar e validar a nitidez durante a visualização mental nos órgãos visuais e cinestésicos (Atienza, Balaguer, \& Merita, 1994). Este instrumento permitiu estabelecer uma relação entre 25 modalidades motoras e habilidades na sua execução, nitidez das imagens e perceção de movimentos (Hall \& Pongrac, 1983), com 18 itens divididos em dois fatores: visuais e cinestésicos, com 9 itens cada fator. Para avaliação da clareza da imagética, foram utilizadas duas subescalas, do tipo Likert com 7 níveis de resposta, que variam entre 1 "muito difícil de ver (ou sentir)" e 7 "muito fácil de ver (ou sentir)", de acordo com a respetiva modalidade de visualização mental realizada. Para melhorar a eficiência de administração do MIQ, Hall e Martin (1997) procederam a uma revisão do MIQ, com o objetivo de reduzir o número de questões, modificar a pontuação dos itens e alterar a formulação semântica do questionário. $\mathrm{O}$ Movement Imagery Questionnaire - Revised (MIQ - R) apresenta oito tarefas para medir a nitidez durante a visualização mental nas modalidades visuais e cinestésicas, quatro na modalidade visual e igual número na modalidade cinestésica. Monsma, Short, Hall, Gregg, e Sullivan (2009) fizeram uma revisão das propriedades psicométricas do MIQ$\mathrm{R}$, apresentando o alfa de Cronbach valores acima de 0.80 , nos dois fatores estudados (modalidade cinestésica e visual), onde concluíram que é um instrumento que pode ser aplicado como uma ferramenta de diagnóstico, de pesquisa para intervenções com o Imagery e de teste para medições como as propostas pelos modelos que guiam a investigação e aplicação prática do Imagery (Applied model of imagery use: Martin et al., 1999; Revised applied model of deliberate imagery use: Cumming \& Williams, 2013). A popularidade do MIQ-R conduziu a uma versão mais recente do questionário Movement Imagery Questionnaire-Revisd Second Edition (MIQ-RS; Gregg, Hall, \& Butler, 2010) desenvolvido especificamente para o contexto da reabilitação.

O MIQ-3 é a versão mais recente do MIQ (Hall \& Pongrac,1983) sendo uma adaptação do MIQR (Hall \& Martin, 1997). É um questionário com 
12 itens para avaliar a habilidade individual de visualização mental de movimentos composto por 3 fatores com quatro itens cada, a visualização mental interna, visualização mental externa e a visualização mental cinestésica proposto por Williams et al. (2012). Os mesmos autores demonstraram que o MIQ-3 é um instrumento que apresenta boas qualidades psicométricas para avaliar os diferentes tipos de habilidades de Imagery, uma vez que, através da análise fatorial confirmatória verificaram valores para a confiabilidade composta acima de 0.7 e da variância média extraída acima de 0.5. Vários estudos utilizaram o MIQ-3, apresentando o alfa de Cronbach valores acima de 0.7 nos três fatores estudados (modalidade cinestésica, visual externa e visual Interna) e no total do instrumento (Debarnot, Abichou, Kalenzaga, Sperduti, \& Piolino, 2015; Nezam, IsaZadeh, Hojati, \& Zadeh, 2014; Williams, Coley, \& Cumming., 2013).

Desta forma, o objetivo deste estudo foi traduzir e validar preliminarmente para a população desportiva portuguesa o Movement Imagery Questionnaire-3.

\section{Participantes}

\section{MÉTODO}

Neste estudo, participaram 176 sujeitos $(\mathrm{N}=$ 176) (sexo masculino $\mathrm{N}=134$; sexo feminino $\mathrm{N}$ = 42) com uma média de idades de 20.08 anos $(\mathrm{SD}=5.94)$. A amostra foi constituída por atletas de futebol $(\mathrm{N}=113)$, basquetebol $(\mathrm{N}=$ 43) e ténis $(\mathrm{N}=20)$ que fossem capazes de realizar os quatro movimentos do MIQ-3 e não tivessem tido contacto anterior com o Imagery.

Quanto à natureza da nossa amostra, podemos afirmar que esta é intencional, por conveniência, uma vez que foi a mais adequada ao tipo de estudo que realizámos e consideramola do tipo não probabilístico, dado que foi selecionada por critérios subjetivos do investigador e de acordo com o objetivo do estudo (Tuckman \& Harper, 2012).

\section{Instrumentos}

O MIQ- 3 (Williams et al., 2012) é um instrumento composto por três subescalas para avaliar as modalidades cinestésica, visual interna e visual externa. São realizados quatro movimentos básicos: levantar o joelho, saltar, movimentar o braço, e dobrar a partir da cintura. Os mesmo quatro movimentos são realizados fisicamente e recriados mentalmente três vezes (em cada uma das modalidades), originando um questionário de 12 itens. Para avaliação da clareza da imagética, utilizaram-se duas subescalas do tipo Likert com 7 pontos de medida, que vão desde "muito difícil de ver (ou sentir)" até "muito fácil de ver (ou sentir)", de acordo com a modalidade de Imagery realizada. Foram fornecidas aos participantes as definições das modalidades cinestésica, visual interna e visual externa antes do preenchimento do questionário. A modalidade visual interna foi definida como "Quando te estás a ver a realizar o movimento de um ponto de vista interno, ou na perspetiva da primeira pessoa, é como se estivesses realmente dentro de ti, realizando e vendo a ação através dos teus olhos". A modalidade visual externa como "Quando te vês a realizar um movimento através de um ponto de vista externo ou da terceira pessoa. Como se te estivesses a ver num DVD". A modalidade cinestésica como "Os sentimentos e sensações que experiencias se estivesses realmente a realizar o movimento". O MIQ-3 demonstrou boa consistência interna para cada fator através da análise fatorial exploratória, verificando-se valores de alfa de Cronbach superiores a 0.7., e da variância média extraída acima de 0.5 . O score do Imageryé obtido através da soma da avaliação da imagem visual interna, externa e do sentido cinestésico, apresentando cada uma das modalidades um score máximo de 28 valores e no total do MIQ-3 de 84 valores. 


\section{Procedimentos}

Para o desenvolvimento da adaptação portuguesa do instrumento de avaliação, seguiuse uma metodologia composta por duas fases: (1) a fase de tradução e adaptação cultural do questionário e (2) a aplicação do método de Análise Fatorial Exploratória do instrumento.

\section{Tradução do questionário}

O objetivo de um processo de validação linguística é obter uma tradução de um instrumento original na língua-alvo, que seja conceitualmente equivalente ao original e facilmente compreendido pelos indivíduos a quem $\mathrm{o}$ questionário traduzido seja administrado. Foi feito um pedido autorização de tradução e validação para a versão Portuguesa ao autor original do MIQ-3 (Williams et al., 2012). O Processo de adaptação cultural do MIQ-3 foi realizado de acordo com a metodologia sequencial (Acquadro, Conway, Hareendran, \& Aaronson, 2012; Beaton, Bombardier, Guillemin, \& Ferraz, 2000; Guillemin, Bombardier, \& Beaton,1993;), utilizando a técnica traduçãoretroversão (translation/back translation technique) (ver Vallerand, 1989). A versão Inglesa do MIQ-3 foi traduzida para Português, de forma independente, por dois tradutores cuja língua materna era o Português, sendo um doutorado na área do desporto e outro na área da linguística. As traduções obtidas foram analisadas num primeiro painel de consenso, por um doutorado da área do desporto e um da área da linguística, do qual resultou a primeira versão preliminar. Esta versão de consenso foi retrovertida para Inglês, de forma independente, por dois tradutores cuja língua materna era o Inglês e que desconheciam a versão original. As traduções e retroversões foram analisadas num segundo painel de consenso e revistas por um doutorado da área do desporto e um da área da linguística, para obter a segunda versão preliminar. Esta versão de consenso foi preenchida por um painel de 20 alunos (10 do género feminino, 10 do género masculino, todos no ensino superior do curso de Desporto e Atividade Física) com média de idades de 19.18 anos $(S D=4.65)$ para verificar se todos os itens do questionário eram adequados, compreensíveis e incluíam todos os conceitos esperados sem qualquer redundância. Os indivíduos foram selecionados de acordo com os critérios utilizados no estudo de validação, mas não participaram no estudo principal. Nenhum dos sujeitos declarou ter tido qualquer dificuldade na compreensão do questionário ou em entender o solicitado e todos consideraram tratar-se de um questionário claro. Na sequência deste painel de indivíduos foi obtida a versão Portuguesa final do MIQ - 3.

\section{Recolha de dados}

Todos os sujeitos ou encarregados de educação dos indivíduos com menos de 18 anos de idade foram devidamente informados sobre o estudo, no que respeita à participação dos seus educandos, desde os objetivos até aos procedimentos, sendo que apenas foram incluídos na amostra, os que deram o seu consentimento, satisfazendo os requisitos e preenchendo o termo de consentimento informado, seguindo a Declaração de Helsínquia (2008). Todas as instruções relativas aos procedimentos foram apresentadas por escrito, para que cada sujeito recebesse as mesmas indicações. O instrumento foi aplicado a todos os participantes sempre em locais e condições semelhantes, numa sala com grupos de número máximo de 5 atletas, onde foram garantidas as condições adequadas para que os atletas pudessem estar concentrados durante a aplicação do questionário. O critério de participação e uniformização da amostra foi a prática desportiva federada há pelo menos dois anos.

Os dados foram recolhidos de forma anónima garantindo-se a confidencialidade dos mesmos, e assegurando-se que não seriam transmitidos individualmente a terceiros.

\section{Análise estatística}

Para a análise de dados, recorremos a uma análise fatorial exploratória (AFE), realizada no SPSS (v.21.0), para determinar o número de fatores a reter, o número de itens que lhes estão associados e a consistência interna dos mesmos.

Para que a AFE tenha utilidade na estimação de fatores comuns deve existir uma correlação elevada entre as variáveis, recorrendo-se à 
medida de adequação da amostragem de KaiserMeyer-Olkin (teste KMO) e o teste de esfericidade de Bartlett, pois permitem aferir a qualidade das correlações de forma a prosseguir ou não com a análise fatorial. $\mathrm{O}$ valor recomendado do teste KMO é que seja superior a 0.6 e o valor do teste de Bartlett seja significativo, sendo essas duas referências cumpridas no nosso estudo (KMO $=.885$; Teste de Bartlett $p=.000$ ) (Worthington \& Whittaker, 2006).

Para a análise de dados, recorremos a uma análise fatorial exploratória (AFE) para determinar o número de fatores a reter, o número de itens que lhes estão associados e a consistência interna dos mesmos. O tipo de rotação adotado foi a rotação oblíqua Promax, uma vez que, de acordo com Preacher e MacCallum (2003, p. 40), o uso do mecanismo de rotação ortogonal Varimax deve ser evitado, visto que, a sua utilização raramente é sustentada, pois os fatores quase nunca são independentes. Para além disso, a rotação oblíqua Promax, é quase sempre a melhor escolha, pois começa com uma análise com uma rotação ortogonal e termina com uma solução oblíqua. De uma forma mais prática, se os fatores forem independentes, a rotação permanece ortogonal, mas se estiverem correlacionados a rotação será oblíqua, sendo esta a razão pela qual optámos por este método de rotação.

Os critérios de determinação da AFE, com base nas recomendações de diversos autores tais como Dimitrov (2012), Hair, Black, Babin, e Anderson (2014), Kaiser (1974), Kahn (2006), Worthington e Whittaker (2006) e operacionalizadas por Cid, Rosado, Alves, e Leitão (2012) foram: Critério de Kaiser; fatores com valor próprio igual ou superior a 1 (eigenvalue $\geq 1.0$ ); os fatores extraídos devem explicar pelo menos $40 \%$ da variância dos resultados; peso fatorial dos itens deve ser igual ou superior a 0.5 (factor loadings $\geq .50$ ). Valores inferiores a 0.30 são inaceitáveis; inexistência de itens com pesos fatoriais com alguma relevância (fator loadings $>$.30) em mais do que um fator. Se isso acontecer e se a diferença entre eles não for significativa (cross-loadings $\leq .15$ ), o item deve ser eliminado. A consistência interna do fator deve ser igual ou superior a 0.70 (alfa de Cronbach $\geq .70$ ). Valores inferiores a 0.60 são inaceitáveis; a consistência interna do fator não deve aumentar se o item for eliminado e por fim, só devem ser retidos os fatores com pelo menos três itens.

\section{RESULTADOS}

Referente à análise descritiva dos resultados (tabela 1), podemos verificar que os sujeitos utilizaram os scores de respostas disponíveis nos itens, tendo o valor médio das respostas de cada um dos itens variado entre $4.94 \pm 1.72$ (item 7) e $5.90 \pm 1.21$ (item 9)

Tabela 1

Estatísticas descritivas das respostas aos itens do MIQ-3

\begin{tabular}{cccccc}
\hline & Mín. - Máx & Média $\pm \mathrm{dp}$ & Assimetria & Curtose & Kolmogorov-Smirnov \\
\hline Item 1 & $1-7$ & $4.97 \pm 1.54$ & -0.69 & -0.37 & $.000^{*}$ \\
Item 2 & $1-7$ & $5.31 \pm 1.45$ & -0.86 & -0.012 & $.000^{*}$ \\
Item 3 & $1-7$ & $5.59 \pm 1.23$ & -1.07 & 0.74 & $.000^{*}$ \\
Item 4 & $1-7$ & $5.33 \pm 1.62$ & -0.89 & -0.14 & $.000^{*}$ \\
Item 5 & $1-7$ & $5.73 \pm 1.23$ & -0.79 & -0.17 & $.000^{*}$ \\
Item 6 & $1-7$ & $5.69 \pm 1.28$ & -1.07 & 0.91 & $.000^{*}$ \\
Item 7 & $1-7$ & $4.94 \pm 1.72$ & -0.69 & -0.69 & $.000^{*}$ \\
Item 8 & $1-7$ & $5.85 \pm 1.26$ & -1.57 & 2.60 & $.000^{*}$ \\
Item 9 & $1-7$ & $5.90 \pm 1.16$ & -1.08 & 0.66 & $.000^{*}$ \\
Item 10 & $1-7$ & $5.34 \pm 1.51$ & -0.79 & -0.29 & $.000^{*}$ \\
Item 11 & $1-7$ & $5.90 \pm 1.21$ & -1.60 & 3.22 & $.000^{*}$ \\
Item 12 & $1-7$ & $5.65 \pm 1.37$ & -1.07 & 0.59 & $.000^{*}$ \\
\hline
\end{tabular}

${ }^{*} p<0.05$

Através do $p$-value do teste de KolmogorovSmirnov constatamos que as respostas nos diversos itens não têm uma distribuição normal $(p<.05)$. Os scores representados apresentam uma distribuição assimétrica negativa (enviesada à esquerda - predominância dos valores mais baixos da variável), os itens da modalidade cinestésica e todos os itens da modalidade visual 
interna um (item 11) têm uma distribuição leptocúrtica (menos achatada que a distribuição normal - valores mais concentrados); os itens da modalidade visual externa têm uma distribuição platicúrtica (mais achatada que a distribuição normal - valores menos concentrados).
Ao analisarmos a tabela 2 verificamos que o alfa de Cronbach apresenta os seguintes valores: no total do MIQ-3 versão Portuguesa um valor de 0.88 , para a modalidade cinestésica 0.79 , para a modalidade visual interna 0.79 e modalidade visual externa 0.79. As correlações entre os itens e os respetivos fatores variam entre 0.76 e 0.83 .

Tabela 2

Análise da Consistência Interna do MIQ-3 versão Portuguesa

\begin{tabular}{ccc}
\hline Miq-3 versão Portuguesa $(\alpha=.88)$ & Correlação Item-Fator & Alfa se Item Eliminado \\
\cline { 2 - 3 } Modalidade Cinestésica $(\alpha=.79)$ & .75 & .78 \\
Item 1 & .76 & .78 \\
Item 4 & .81 & .76 \\
Item 7 & .83 & .79 \\
Item 10 & & .76 \\
Item 2 & .82 & .78 \\
Item 5 & .76 & .77 \\
Item 8 & .81 & .78 \\
Item 11 & .77 & .78 \\
Modalidade Visual Interna $(\alpha=.79)$ & & .77 \\
Item 3 & .77 & .78 \\
Item 6 & .81 & .77 \\
Item 9 & .76 & \\
Item 12 & .80 & \\
\hline
\end{tabular}

Os resultados relativos à estrutura do questionário com recurso à AFE são apresentados na tabela 3. Existe uma estrutura de 2 fatores com valores próprios acima de 1 , correspondente a 5.40 para a modalidade visual externa (item 3, 6, 9 e 12), 1.26 para a modalidade cinestésica (item 1, 4, 7 e 10), e um fator com valores próprios abaixo de 1 , correspondente 0.92 para a modalidade visual interna (item 2, 5, 8 e 11), que justificam no seu conjunto $63.14 \%$ da variância total dos resultados.

Relativamente aos resultados das comunalidades, todas atingiram valores aceitáveis $(>.50)$, o que indica que uma boa parte da variância dos resultados de cada item é explicada pela solução fatorial encontrada.

Referente aos pesos fatoriais dos itens nos respetivos fatores e tendo em conta a matriz padrão do quadro 3, embora os resultados da matriz de configuração sejam idênticos, todos apresentam valores acima de 0.5 com exceção dos itens 1 e 11 que apresentam valores acima de 0.45. Assim, todos os itens têm pesos fatoriais que variam entre 0.45 e 0.91 (fator "modalidade visual externa": entre 0.48 e 0.84 ; fator "modalidade cinestésica": entre 0.51 e 0.87 ; fator "modalidade visual interna: entre 0.45 e 0.91 ).

Ao analisar os pesos fatoriais dos itens em todos os fatores, verificamos a existência de um cross-loading dos itens 1 e 11, ou seja, estes itens apresentam um peso fatorial relevante em dois fatores. O item 1 no fator "modalidade cinestésica" apresenta um valor 0.51 e no fator da "modalidade visual interna" um valor de 0.46; o item 11 no fator "modalidade visual externa" 0.48 e no fator "modalidade visual interna" 0.45 . O item 1 na matriz padrão, apesar do crossloading, exibe o valor mais alto no fator onde em teoria deveria ter mais peso (modalidade cinestésica), o mesmo não acontecendo com o item 11 . 
Tabela 3

Análise fatorial exploratória (com rotação oblíqua Promax) do MIQ-3 versão Portuguesa

\begin{tabular}{|c|c|c|c|c|c|c|c|}
\hline & \multirow[b]{2}{*}{ Comunalidades } & \multicolumn{3}{|c|}{ Matriz Padrão } & \multicolumn{3}{|c|}{ Matriz de Estruturas } \\
\hline & & Fator & Fator & Fator & Fator & Fator & Fator Visual \\
\hline Item 1 & .59 & & .51 & .46 & & .67 & .64 \\
\hline Item 2 & .58 & & & .59 & & & .73 \\
\hline Item 3 & .63 & .84 & & & .79 & & \\
\hline Item 4 & .68 & & .80 & & & .79 & \\
\hline Item 5 & .72 & & & .91 & & & .84 \\
\hline Item 6 & .65 & .76 & & & .81 & & \\
\hline Item 7 & .69 & & .76 & & & .79 & \\
\hline Item 8 & .62 & & & .64 & & & .76 \\
\hline Item 9 & .52 & .67 & & & .72 & & \\
\hline Item 10 & .77 & & .87 & & & .87 & \\
\hline Item 11 & .55 & .48 & & .45 & .68 & & .62 \\
\hline \multirow[t]{2}{*}{ Item 12} & .58 & .64 & & & .75 & & \\
\hline & & Modalidac & al Externa & Modalida & nestésica & Modalida & Visual Interna \\
\hline \multicolumn{2}{|c|}{ Valor Próprio } & \multicolumn{2}{|c|}{5.40} & \multicolumn{2}{|c|}{1.26} & \multicolumn{2}{|c|}{0.92} \\
\hline \multicolumn{2}{|c|}{ \% Variância } & \multicolumn{2}{|c|}{44.99} & \multicolumn{2}{|c|}{10.53} & \multicolumn{2}{|c|}{7.62} \\
\hline
\end{tabular}

\section{DISCUSSÃO}

O presente estudo teve como objetivo traduzir e validar preliminarmente o MIQ-3 realizado de acordo com a metodologia sequencial (Beaton et al., 2000; Guillemin et al.,1993) e a determinação das qualidades psicométricas através de uma análise fatorial exploratória do modelo que a suporta. Após a tradução do questionário, a análise fatorial exploratória mostra-nos que o modelo proposto por Williams et al. (2012), constituído por três fatores (modalidade cinestésica, visual interna e visual externa), com quatro itens cada, apresenta qualidades psicométricas que comprovam a adequação da adaptação efetuada, demonstrando que a sua estrutura fatorial possui índices bastante aceitáveis. A adaptação de uma escala já existente tem a vantagem de permitir comparar resultados de vários estudos uma vez que os resultados entre culturas podem ser comparados, evitandose a excessiva proliferação de instrumentos sobre os mesmos constructos (Cid, Rosado, Alves, \& Leitão, 2012).

O número de sujeitos utilizados no nosso estudo respeita o rácio de 10:1 ( $\mathrm{n}^{\circ}$ de sujeitos por cada item do questionário), sendo o número que gera mais consenso na literatura (Dimitrov, 2012; Hair, Black, Babin, \& Anderson, 2014; Kahn, 2006; Worthintgton \& Whittaker, 2006). No questionário apresentado seriam necessários no mínimo 120 sujeitos $(n=176)$.

Os valores dos itens disponíveis apresentam uma distribuição assimétrica negativa, os itens da modalidade cinestésica e todos os itens da modalidade visual interna menos um (item 11) têm uma distribuição leptocúrtica (menos achatada que a distribuição normal - valores mais concentrados); os itens da modalidade visual externa têm uma distribuição platicúrtica (mais achatada que a distribuição normal - valores menos concentrados), revelando que os participantes apresentam uma tendência para os valores mais baixos das variáveis e valores mais concentrados (Hair et al., 2014; Härdle \& Simar, 2015).

Verificamos que o alfa de Cronbach apresenta bons valores de consistência interna no total do questionário e nos seus três fatores, estando todos acima de 0.6 , sendo este o valor mínimo de referência para a análise fatorial exploratória (Hair et al., 2014). Nas correlações entre itens e respetivos fatores temos um bom sinal de consistência interna, tendo todas as correlações um valor de 0.79. Segundo Hair et al., (2014), todas as correlações item-fator acima de 0.50 podem ser consideradas boas.

$\mathrm{Na} \mathrm{AFE}$, referente à análise de constructo, o teste do critério de Kaiser $(\mathrm{KMO}=0.885)$ indica que a análise de componentes principais pode ser realizada, e o teste de esfericidade (Teste de Bartlett $p=.000$ ) diz-nos que as variáveis são correlacionáveis. Estes valores permitem aferir a qualidade das correlações uma vez que o valor de KMO é superior a 0.6 e o valor do teste de Bartlett é estaticamente significativo (Ho, 2014; Worthington \& Whittaker, 2006). 
O resultado da análise da solução inicial pela AFE, ao apontar que existe uma estrutura de 2 fatores com valores próprios acima de 1 , correspondente a 5.40 para a modalidade visual externa (item 3, 6, 9 e 12), 1.26 para a modalidade cinestésica (item 1, 4, 7 e 10), e um fator com valores próprios abaixo de 1 , correspondente 0.92 para a modalidade visual interna (item 2, 5, 8 e 11), reflete instabilidade de um fator. Contudo, segundo Hair, Black, Babin, Anderson, e Tatham (2006) quando os valores médios extraídos, não são superiores a 1 , significa que muita variância foi perdida/não explicada. Dessa forma, sustentamo-nos na variância acumulada para determinar quantos fatores devem ser extraídos, devendo esta ser fixada em $60 \%$. No nosso estudo, esta apresentou uma variância acumulada de $63.14 \%$. Por esta razão e porque a teoria subjacente ao instrumento original refere três fatores (Cid et al., 2012) aceitamos o valor de 0.92. no fator modalidade visual interna.

Quanto às comunalidades da matriz de configuração, que é a mais utilizada na investigação aplicada (Brown, 2006), onde são indicados apenas os pesos fatoriais relevantes e considerados como valor mínimo para serem interpretados de 0.5 , mostram-nos que todos os valores cumprem o critério estipulado (Worthington \& Whittaker,2006).

No que concerne aos pesos fatoriais dos itens nos respetivos fatores e tendo em conta o tamanho da amostra $(n=176)$ todos apresentam valores acima de 0.5 (Hair et al., 2014), exceto os itens 1 e 11 , todos os restantes variam entre 0.51 e 0.91. Dessa forma, tomando em consideração o número de participantes do nosso estudo podemos aceitar como suficientes (ie. significativos) os pesos fatoriais acima de 0.45 (Hair et al., 2014), considerando que nenhum item deve ser eliminado.

Por último, relativamente aos pesos fatoriais dos itens e todos os fatores, verificamos a existência de cross-loadings dos itens 1 e 11, apresentando um peso fatorial relevante em dois fatores. O item 1 na matriz padrão, apesar do cross-loading, exibe o valor mais alto no fator onde em teoria deveria ter mais peso (modalidade cinestésica). No entanto, conforme se pode verificar na tabela 2 , a eliminação de qualquer um destes dois itens (item 1 e 11) não melhora o coeficiente alfa de Cronbach dos fatores a que eles estão associados, logo a sua eliminação parece não contribuir para melhoria do modelo. Segundo Ho (2014) quando examinamos a formação das variáveis face à sua validade, estas podem ser atribuídas aos fatores que são mais conceptuais/lógicos na sua representação. Worthington e Whittaker (2006) também aconselham alguma prudência em usar este critério para justificar a eliminação dos itens até que seja encontrada uma solução final.

\section{CONCLUSÕES}

Os objetivos deste estudo consistiram no desenvolvimento de uma versão portuguesa do Movement Imagery Questionnaire- 3 (MIQ - 3; William et al., 2012). Numa primeira fase verificou-se que os procedimentos de tradução e adaptação originaram uma versão Portuguesa do MIQ - 3 semelhante à versão original. Numa segunda fase verificamos que no seu conjunto, as suas qualidades psicométricas comprovam a sua adequação da adaptação efetuada, demonstrando que a sua estrutura fatorial possui índices bastante aceitáveis para a sua validação. A análise da consistência interna no total do questionário e dos seus três fatores foi confirmada pelos valores dos coeficientes de alfa de Cronbach. A validade do questionário na versão portuguesa foi confirmada pela análise fatorial exploratória, tendo-se mantido os três fatores distintos (modalidade cinestésica, visual interna e visual externa) propostos pelos autores na validação da escala original. Uma das limitações do nosso estudo foi a existência de um fator com valor próprio inferior a 1 , o que deve ser tomado em conta em futuras investigações. Apesar do questionário apresentar caraterísticas psicométrica adequadas, consideramos fundamental a continuação dos estudos que confirmem a validade fatorial deste instrumento de medida, tendo em conta que encontramos cross-loadings em duas variáveis sugerimos complementar este estudo efetuando uma análise fatorial confirmatória do questionário. 


\begin{abstract}
Agradecimentos:
Centro Interdisciplinar de Línguas, Cultura e Educação do Instituto Politécnico de Castelo Branco
\end{abstract}

\section{Conflito de Interesses:}

Nada a declarar.

Financiamento:

Nada a declarar

\section{REFERÊNCIAS}

Acquadro, C., Conway, K., Hareendran, A., Aaronson, N., \& European Regulatory Issues and Quality of Life Assessment (ERIQA) Group. (2008). Literature review of methods to translate healthrelated quality of life questionnaires for use in multinational clinical trials. Value in Health: The Journal of the International Society for Pharmacoeconomics and Outcomes Research, 11(3), 509-521. https://doi.org/10.1111/j.15244733.2007.00292.x

Atienza, F., Balaguer, I., \& Garcia-Merita, M. L. (1994). Factor analysis and reliability of the Movement Imagery Questionnaire. Perceptual and Motor Skills, 78(3 Pt 2), 1323-1328. https://doi.org/10.2466/pms.1994.78.3c.1323

Beaton, D., Bombardier, C., Guillemin, F., \& Ferraz, M. (2000). Guidelines for the process of crosscultural adaptation of self-report measures. Spine, 25(24), 3186-3191.

Brown, T. (2006). Confirmatory factor analysis for applied research. New York: The Guiford Press.

Cid, L., Rosado, A., Alves, J. \& Leitão, J. (2012). Tradução e Validação de Questionários em Psicologia do Desporto e Exercício. In A. Rosado, I. Mesquita \& C. Colaço (Ed.), Métodos e técnicas de Investigação Qualitativa (pp.29-64). Lisboa: Edições FMH.

Cumming, J., \& Williams, S. (2013). Introducing the revised applied model of deliberate imagery use for sport, dance, exercise, and rehabilitation. Movement \& Sport Sciences - Science \& Motricité, 82, 69-81. doi: 10.1051/sm/2013098

Cumming, J., \& Ste-Marie, D. (2001). The cognitive and motivational effects of imagery training: A matter of perspective. The Sport Psychologist, 15, 276-288

Debarnot, U., Abichou, K., Kalenzaga, S., Sperduti, M., \& Piolino, P. (2015). Variable motor imagery training induces sleep memory consolidation and transfer improvements. Neurobiology of Learning and Memory, 119, 85-92. https://doi.org/10.1016/j.nlm.2014.12.010

Declaração de Helsínquia da Associação Médica Mundial (2008). Princípios Éticos para a Investigação Médica em Seres Humanos. Seul.

Dimitrov, D. (2012). Statistical Methods for Validation of Assessment Scale Data in
Counselling and Related Fields. Alexandria: Wiley.

Gregg, M., Hall, C. \& Butler, A. (2010). The MIQ-RS: A suitable option for examining movement imagery ability. Evidence-Based Complementary and Alternative Medicine, 7(2), 249-257. doi: 10.1093/ecam/nem170

Guillemin, F., Bombardier, C., \& Beaton, D. (1993). Cross-cultural adaptation of health-related quality of life measures: literature review and proposed guidelines. Journal of Clinical Epidemiology, 46(12), 1417-1432. https://doi.org/10.1016/0895-4356(93)90142-N

Hair, J., Black, W., Babin, B. \& Anderson, R. (2014). Multivariate Data Analysis ( $7^{\mathrm{a}}$ ed.). Harlow: Pearson New Internacional Edition.

Hair, J., Black, W., Babin, B., Anderson, R. \& Tatham, R. (2006). Multivariate Data Analysis (6 $6^{\mathrm{a}}$ ed.). New Jersey: Pearson Education, Inc.

Hall, C. (2001). Imagery in sport and exercise. In R. N. Singer, H. Hausenblas, \& C. Janelle (Ed.), Handbook of sport psychology ( $2^{\mathrm{a}}$ ed., pp. 529549). New York: John Wiley \& Sons.

Hall, C. R., \& Martin, K. A. (1997). Measuring movement imagery abilities: A revision of the Movement Imagery Questionnaire. Journal of Mental Imagery, 21(1-2), 143-154.

Hall, C., \& Pongrac, J. (1983). Movement imagery questionnaire. London, Ontario: University of Western Ontario.

Hall, C., Mack, D., Pavio, A. \& Hausenblas, H. (1998). Imagery use by athletes: Development of the Sport Imagery Questionnaire. International Journal of Sport Psychology, 29(1), 73-89.

Härdle, W \& Simar, L. (2015). Applied Multivariate Statistical Analysis (4a ed.). London: Springer. Doi:10.1007/978-3-662-45171-7

Ho R. (2014). Handbook of Univariate and Multivariate Data Analysis with IBM SPSS (2 ${ }^{\mathrm{a}}$ Ed.). New York:CRC Press. doi: 0.1080/02664763.2015.1015811

Holmes, P., \& Calmels, C. (2008). A neuroscientific review of imagery and observation use in sport. Journal of Motor Behavior, 40(5), 433-445. https://doi.org/10.3200/JMBR.40.5.433-445

Isaac, A., Marks, D. F., \& Russell, D. G. (1986). An instrument for assessing imagery of movement: The Vividness of Movement Imagery Questionnaire (VMIQ). Journal of Mental Imagery, 10(4), 23-30.

Kahn, J. H. (2006). Factor Analysis in Counseling Psychology Research, Training, and Practice Principles, Advances, and Applications. The Counseling Psychologist, 34(5), 684-718. https://doi.org/10.1177/0011000006286347

Kaiser, H. F. (1974). An index of factorial simplicity. Psychometrika, 39(1), 31-36. https://doi.org/10.1007/BF02291575

Martin, K. A., Moritz, S. E., \& Hall, C. R. (1999). Imagery Use in Sport: A Literature Review and Applied Model. The Sport Psychologist, 13(3), 245-268. https://doi.org/10.1123/tsp.13.3.245 
158 | PA Mendes, DA Marinho, JD Petrica, P Silveira, D Monteiro, L Cid

Monsma, E. V., Short, S. E., Hall, C. R., Gregg, M., \& Sullivan, P. (2009). Psychometric Properties of the Revised Movement Imagery Questionnaire (MIQ-R). Journal of Imagery Research in Sport and Physical Activity, 4(1), 1-12. https://doi.org/10.2202/1932-0191.1027

Morris, T., Spittle, M. \& Watt, A. (2005) Imagery in Sport (Chap. 10. Technical Aids to Imagery, pp. 237-266). Champaign, IL: Human Kinetics.

Nezam, S., IsaZadeh, H., Hojati, A. \& Zadeh, Z. (2014). Comparison Ability of Movement Imagery perspectives in Elite, Sub-Elite and NonElite Athletes. International Research Journal of Applied and Basic Sciences, 8(6), 712-716.

Preacher, K. \& MaCallum, R. (2003). Repairing Tom Swift's electric factor analysis machine. Understanding Statistics, 2(1), 13-43.

Tuckman, B. \& Harper, B. (2012). Conducting Educational Research (6 $6^{\mathrm{a}}$ ed.). United Kingdom: Rowman Littlefield Publishers,Inc.
Vallerand, R. (1989). Vers une méthodologie de validation transculturelle de questionnaires psychologiques: implications pour la recherche en langue française. Psychologie Canadienne, 30(4), 662-689. doi:10.1037/h0079856

Williams, S. E., Cooley, S. J., \& Cumming, J. (2013). Layered stimulus response training improves motor imagery ability and movement execution. Journal of Sport \& Exercise Psychology, 35(1), 60-71.

Williams, S. E., Cumming, J., Ntoumanis, N., NordinBates, S. M., Ramsey, R., \& Hall, C. (2012). Further validation and development of the movement imagery questionnaire. Journal of Sport \& Exercise Psychology, 34(5), 621-646.

Worthington, R. L., \& Whittaker, T. A. (2006). Scale Development Research A Content Analysis and Recommendations for Best Practices. The Counseling Psychologist, 34(6), 806-838. https://doi.org/10.1177/0011000006288127

Todo o conteúdo da revista Motricidade está licenciado sob a Creative Commons, exceto quando especificado em contrário e nos conteúdos retirados de outras fontes bibliográficas. 